Asia Pacific Journal of Mathematics, Vol. 5, No. 2 (2018), 208-218

ISSN 2357-2205

\title{
FUZZY BI-INTERIOR IDEALS OF SEMIGROUPS
}

\author{
M. MURALI KRISHNA RAO \\ Department of Mathematics, GIT, GITAM University, Visakhapatnam- 530 045, A.P., INDIA \\ Email address: mmarapureddy@gmail.com
}

Received May 11, 2018

\begin{abstract}
In this paper,we introduce the notion of a fuzzy bi-interior ideal of semigroup and we characterize the regular semigroup in terms of a fuzzy bi-interior ideal of a semigroup. 2010 Mathematics Subject Classification. 03E72,20M12.
\end{abstract}

Key words and phrases. semigroup; regular semigroup; bi-quasi ideal; bi-interior ideal; fuzzy bi-quasi ideal; fuzzy bi-interior ideal.

\section{INTRODUCTION}

In 2017, as a further generalization of ideals in semigroups, Murali Krishna Rao [16] introduced the concept of a bi-interior ideal for a semigroup as a generalization of ideal,left(right) ideal, bi-ideal, interior ideal and quasi ideal. Semigroup, as the basic algebraic structure was used in the areas of theoretical computer science as well as in the solutions of graph theory, optimization theory and in particular for studying automata, coding theory and formal languages. Ideals play an important role in advance studies of algebraic structures. Generalization of ideals in algebraic structures is necessary for further study of algebraic structures. Many mathematicians proved important results and charecterization of algebraic structures by using the concept and the properties of generalization of ideals in algebraic structures. The notion of ideals was introduced by Dedekind for the theory of algebraic numbers, was generalized by Noether for associative rings. The one and two sided ideals introduced by her, are still central concepts in ring theory and the notion of an one sided ideal of any algebraic structure is a generalization of notion of an ideal.

In 1952, the concept of bi-ideals was introduced by Good and Hughes [1] for semigroups. The notion of bi-ideals in rings and semirings were introduced by Lajos and Szasz [6,7].Quasi ideals are generalization of right ideals and left ideals whereas bi-ideals are generalization of quasi ideals. In 1956,Steinfeld[25] first introduced the notion of quasi ideals for semigroups and then for rings. Iseki $[2,3]$ introduced the concept of quasi ideal for a semiring. Quasi ideals in $\Gamma$-semirings were studied by Jagtap and Pawar [4]. Shabir et al. [24] studied ideals

(c)2018 Asia Pacific Journal of Mathematics 
in semirings.In 1995, Murali Krishna Rao [13,14,15] introduced the notion of $\Gamma$-semiring as a generalization of semiring.In 2016,Murali Krishna Rao [11,12,17] introduced the notion of left (right) bi-quasi ideal of semiring, $\Gamma$-semiring, $\Gamma$-semigroup and studied the properties of left bi-quasi ideals. We characterize the left bi-quasi simple $\Gamma$-semiring and regular $\Gamma$-semiring using left bi-quasi ideals of $\Gamma$-semiring.

The fuzzy set theory was developed by Zadeh [26] in 1965.The fuzzification of algebraic structure was introduced by Rosenfeld [17] and he introduced the notion of fuzzy subgroups in 1971. Many papers on fuzzy sets appeared showing the importance of the concept and its applications to logic, set theory, group theory, ring theory, real analysis, topology, measure theory etc. In 1982, Liu [8] defined and studied fuzzy subrings as well as fuzzy ideals in rings. Mandal [9] studied fuzzy ideals and fuzzy interior ideals in an ordered semiring. Murali Krishna Rao [10, 19,21]studied fuzzy soft $\Gamma$-semiring, T-fuzzy ideals,fuzzy soft biideal,fuzzy soft quasi-ideal and fuzzy soft interior ideal over ordered $\Gamma$ - semirings and fuzzy soft $k$-ideal over $\Gamma$-semiring.

In this paper,we introduce the notion of a fuzzy bi-interior ideal of semigroup and we characterize the regular semigroup in terms of fuzzy bi-quasi ideals of semigroup.

\section{Preliminaries}

In this section, we will recall some of the fundamental concepts and definitions, which are necessary for this paper.

Definition 2.1. A semigroup is an algebraic system $(M,$.$) consisting of a non-empty set M$ together with an associative binary operation ". .

Definition 2.2. A subsemigroup $T$ of a semigroup $M$ is a non-empty subset $T$ of $M$ such that $T T \subseteq T$.

Definition 2.3. A non-empty subset $T$ of a semigroup $M$ is called a left (right) ideal of $M$ if $M T \subseteq T(T M \subseteq T)$.

Definition 2.4. A non-empty subset $T$ of a semigroup $M$ is called an ideal of $M$ if it is both a left ideal and a right ideal of $S$.

Definition 2.5. A non-empty $Q$ of a semigroup $M$ is called a quasi ideal of $M$ if $Q M \cap M Q \subseteq$ $Q$.

Definition 2.6. A subsemigroup $T$ of a semigroup $M$ is called a bi-ideal of $M$ if $T M T \subseteq T$.

Definition 2.7. A subsemigroup $T$ of a semigroup $M$ is called an interior ideal of $M$ if $M T M \subseteq T$. 
Definition 2.8. An element $a$ of a semigroup $M$ is called a regular element if there exists an element $b$ of $M$ such that $a=a b a$. A semigroup $M$ is called a regular semigroup if every element of $M$ is a regular element.

Definition 2.9. Let $M$ be a non-empty set. A mapping $\mu: M \rightarrow[0,1]$ is called a fuzzy subset of $M$.

Definition 2.10. If $\mu$ is a fuzzy subset of $M$, for $t \in[0,1]$, then the set $\mu_{t}=\{x \in M \mid \mu(x) \geq$ $t\}$ is called a level subset of $M$ with respect to a fuzzy subset $\mu$.

Definition 2.11. Let $M$ be a non-empty set. A fuzzy subset $\mu: M \rightarrow[0,1]$ is a non-empty fuzzy subset if $\mu$ is not a constant function.

Definition 2.12. Let $M$ be a non-empty set. For any two fuzzy subsets $\lambda$ and $\mu$ of $M$, $\lambda \subseteq \mu$ means $\lambda(x) \leq \mu(x)$ for all $x \in M$.

Definition 2.13. Let $M$ be a non-empty set and $A$ be a non-empty subset of $M$. The characteristic function of $A$ is a fuzzy subset of $M$ is defined by $\chi_{A}(x)= \begin{cases}1, & \text { if } x \in A ; \\ 0, & \text { if } x \notin A .\end{cases}$

Definition 2.14. Let $M$ be a non-empty set, $f$ and $g$ be fuzzy subsets of $M$. Then $f \cup g, f \cap g$ are fuzzy subsets of $S$ defined by $f \cup g(x)=\max \{f(x), g(x)\}, f \cap g(x)=\min \{f(x), g(x)\}$ for all $x \in S$. And $f \circ g$ is defined by

$$
f \circ g(z)=\left\{\begin{array}{ll}
\sup _{z=x y, x, y \in S} & \{\min \{f(x), g(y)\}\}, \\
0 & \text { otherwise }
\end{array}, \text { for all } z \in M .\right.
$$

Definition 2.15. A fuzzy subset $\mu$ of a semigroup $M$ is called

(i) a fuzzy subsemigroup of $M$ if $\mu(x y) \geq \min \{\mu(x), \mu(y)\}$, for all $x, y \in M$.

(ii) a fuzzy left (right) ideal of $M$ if $\mu(x y) \geq \mu(y)(\mu(x))$, for all $x, y \in M$.

(iii) a fuzzy ideal of $M$ if $\mu(x y) \geq \max \{\mu(x), \mu(y)\}$, for all $x, y \in M$.

(iv) a fuzzy left (right)ideal if $\chi_{M} \circ \mu \subseteq \mu\left(\mu \circ \chi_{M} \subseteq \mu\right)$,

(iv) a fuzzy bi-ideal of $M$ if $\mu \circ \chi_{M} \circ \mu \subseteq \mu$,

(vi) a fuzzy quasi -ideal of $M$ if $\mu \circ \chi_{M} \cap \chi_{M} \circ \mu \subseteq \mu$,

Definition 2.16. Let $M$ be a semigroup. A non-empty subset $L$ of $M$ is said to be left (righti) bi-quasi ideal of $M$ if $L$ is a subsemigroup of $M$ and $M L \cap L M L \subseteq L(L M \cap L M L \subseteq$ $L)$.

Definition 2.17. Let $M$ be a semigroup. A non-empty subset $L$ of $M$ is said to be bi-quasi ideal of $M$ if $L$ is a subsemigroup of $M, M L \cap L M L \subseteq L$ and $L M \cap L M L \subseteq L$. 
Theorem 2.18. [16] Let $M$ be a regular semigroup. Then $B$ is a bi-interior ideal of $M$ if and only if $M B M \cap B M B=B$, for all bi-interior ideals $B$ of $M$.

Definition 2.19. [16] Let $M$ be a semigroup. A non-empty subset $L$ of $M$ is said to be bi-interior ideal of $M$ if $L$ is a subsemigroup of $M$ and $M L M \cap L M L \subseteq L$

Theorem 2.20. [16] Let $M$ be a semigroup. Then the following are hold.

(1) Every left ideal is a bi-interior ideal of $M$.

(2) Every right ideal is a bi-interior ideal of $M$.

(3) Every quasi ideal is bi-interior ideal of $M$.

(4) Arbitrary intersection of bi-interior of $M$ is also bi-interior ideal of $M$.

(5) Every ideal is a bi-interior ideal of $M$.

(6) If $B$ is a bi-interior ideal of $M$ then $B M$ and $M B$ are bi-interior ideals of $M$.

\section{FUZZY BI-INTERIOR IDEALS OF SEMIGROUPS}

In this section, we introduce the notion of a fuzzy bi-interior ideal as a generalization of a fuzzy bi-ideal of semigroup $M$ and a fuzzy interior ideal of a semigroup. We study the properties of fuzzy bi-interior ideals of semigroups

Definition 3.1. A fuzzy subset $\mu$ of a semigroup $M$ is called a fuzzy bi-interior ideal if $\chi_{M} \circ \mu \circ \chi_{M} \cap \mu \circ \chi_{M} \circ \mu \subseteq \mu$

Example 3.2. Let $Q$ be the set of all rational numbers, $M=\left\{\left(\begin{array}{ll}a & b \\ 0 & c\end{array}\right) \mid a, b, c \in Q\right\}$ Binary operation is defined on $\mathrm{M}$ as the usual matrix multiplication and

$A=\left\{\left(\begin{array}{ll}a & 0 \\ 0 & b\end{array}\right) \mid a, 0 \neq b \in Q\right\}$. Then $M$ is a semigroup and $A$ is a bi-interior ideal but not a bi-ideal and an interior ideal of a semigroup $M$.

Define $\mu: M \rightarrow[0,1]$ such that $\mu(x)== \begin{cases}1 & \text { if } x \in A, \\ 0, & \text { otherwise. }\end{cases}$

Then $\mu$ is a fuzzy bi-interior ideal of $M$.

Theorem 3.3. Every fuzzy left ideal of a semigroup $M$ is a fuzzy bi-interior ideal of $M$. 
Proof. Let $\mu$ be a fuzzy left ideal of the semigroup $M$ and $x \in M$.

$$
\begin{aligned}
\chi_{M} \circ \mu(x) & =\sup _{x=a b}\left\{\min \left\{\chi_{M}(a), \mu(b)\right\}\right\} \\
& =\sup _{x=a b}\{\min \{1, \mu(b)\}\} \\
& =\sup _{x=a b}\{\mu(b)\} \\
& \leq \sup _{x=a b}\{\mu(a b)\} \\
& =\sup _{x=a b}\{\mu(x)\} \\
& =\mu(x) \\
\Rightarrow \chi_{M} \circ \mu(x) & \leq \mu(x) . \\
\mu \circ \chi_{M} \circ \mu(x) & =\sup _{x=u v s}\left\{\min \left\{\mu(u), \chi_{M} \circ \mu(v s)\right\}\right\} \\
& \leq \sup _{x=u v s}\{\min \{\mu(u), \mu(v s)\}\} \\
& =\mu(x) .
\end{aligned}
$$

$$
\text { Now } \begin{aligned}
\chi_{M} \circ \mu \circ \chi_{M} \cap \mu \circ \chi_{M} \circ \mu(x) & =\min \left\{\chi_{M} \circ \mu \circ \chi_{M}(x), \mu \circ \chi_{M} \circ \mu(x)\right\} \\
& \leq \min \left\{\chi_{M} \circ \mu \circ \chi_{M}(x), \mu(x)\right\} \\
& \leq \mu(x)
\end{aligned}
$$

Therefore $\chi_{M} \circ \mu \circ \chi_{M} \cap \mu \circ \chi_{M} \circ \mu \subseteq \mu$.

Hence $\mu$ is a fuzzy bi-interior ideal of the semigroup $M$.

Theorem 3.4. Every fuzzy right ideal of a semigroup $M$ is a fuzzy bi-interior ideal of $M$.

Proof. Let $\mu$ be a fuzzy right ideal of the semigroup $M$ and $x \in M$.

$$
\begin{aligned}
\mu \circ \chi_{M}(x) & =\sup _{x=a b}\left\{\min \left\{\mu(a), \chi_{M}(b)\right\}\right\} \\
& =\sup _{x=a b}\{\mu(a)\} \\
& \leq \sup _{x=a b}\{\mu(a b)\} \\
& =\mu(x) .
\end{aligned}
$$




$$
\begin{aligned}
& \text { Therefore } \mu \circ \chi_{M}(x) \leq \mu(x) \\
& \text { Now } \mu \circ \chi_{M} \circ \mu(x)=\sup _{x=u v s}\left\{\min \left\{\mu \circ \chi_{M}(u v), \mu(s)\right\}\right\} \\
& \leq \sup _{x=u v s}\{\min \{\mu(u v), \mu(s)\}\} \\
& =\mu(x) \text {. } \\
& \text { Now } \chi_{M} \circ \mu \circ \chi_{M} \cap \mu \circ \chi_{M} \circ \mu(x)=\min \left\{\chi_{M} \circ \mu \circ \chi_{M}(x), \mu \circ \chi_{M} \circ \mu(x)\right\} \\
& \leq \min \left\{\chi_{M} \circ \mu \circ \chi_{M}(x), \mu(x)\right\} \\
& \leq \mu(x) \text {. }
\end{aligned}
$$

Therefore $\chi_{M} \circ \mu \circ \chi_{M} \cap \mu \circ \chi_{M} \circ \mu \subseteq \mu$.

Hence $\mu$ is a fuzzy bi-interior ideal of the semigroup $M$.

Corollary 3.5. Every fuzzy ideal of a semigroup $M$ is a fuzzy bi-interior ideal of $M$.

Theorem 3.6. Let $M$ be a semigroup and $\mu$ be a non-empty fuzzy subset of $M$. A fuzzy subset $\mu$ is a fuzzy bi-interior ideal of a semigroup if and only if the level subset $\mu_{t}$ of $\mu$ is a bi-interior ideal of a semigroup $M$ for every $t \in[0,1]$, where $\mu_{t} \neq \phi$.

Proof. Let $M$ be a semigroup and $\mu$ be a non-empty fuzzy subset of $M$. Suppose $\mu$ is a fuzzy bi-interior ideal of the semigroup $M, \mu_{t} \neq \phi, t \in[0,1]$

Let $x \in M \mu_{t} M \cap \mu_{t} M \mu_{t}$. Then $x=b a u=c d e$, where $b, u, d \in M, a, c, e \in \mu_{t}$. Then

$\chi_{M} \circ \mu \circ \chi_{M}(x) \geq t$ and $\mu \circ \chi_{M} \circ \mu(x) \geq t$

$\Rightarrow \mu(x) \geq t$

Therefore $x \in \mu_{t}$.

Hence $\mu_{t}$ is a bi-interior ideal of the semigroup $M$.

Conversely suppose that $\mu_{t}$ is a bi-interior ideal of the semigroup $M$, for all $t \in \operatorname{Im}(\mu)$.

Let $x, y \in M, \mu(x)=t_{1}, \mu(y)=t_{2}$ and $t_{1} \geq t_{2}$. Then $x, y \in \mu_{t_{2}}$.

We have $M \mu_{l} M \cap \mu_{l} M \mu_{l} \subseteq \mu_{t}$, for all $l \in \operatorname{Im}(\mu)$.

Suppose $t=\min \{\operatorname{Im}(\mu)\}$. Then $M \mu_{t} M \cap \mu_{t} M \mu_{t} \subseteq \mu_{t}$.

Therefore $\chi_{M} \circ \mu \circ \chi_{M} \cap \mu \circ \chi_{M} \circ \mu \subseteq \mu$.

Hence $\mu$ is a fuzzy bi-interior ideal of the semigroup $M$.

Theorem 3.7. Let I be a non-empty subset of a semigroup $M$ and $\chi_{I}$ be the characteristic function of $I$. Then $I$ is a bi-interior ideal of a semigroup $M$ if and only if $\chi_{I}$ is a fuzzy bi-interior ideal of a semigroup $M$.

Proof. Let $I$ be a non-empty subset of the semigroup $M$ and $\chi_{I}$ be the characteristic function of $I$. Suppose $I$ is a bi-interior ideal of the semigroup $M$. Obviously $\chi_{I}$ is a fuzzy subsemigroup 
of $M$. We have $M I M \cap I M I \subseteq I$. Then

$$
\begin{aligned}
\chi_{M} \circ \chi_{I} \circ \chi_{M} \cap \chi_{I} \circ \chi_{M} \circ \chi_{I} & =\chi_{M I M} \cap \chi_{I M I} \\
& =\chi_{M I M \cap I M I} \\
& \subseteq \chi_{I} .
\end{aligned}
$$

Therefore $\chi_{I}$ is a fuzzy bi-interior ideal of the semigroup $M$.

Conversely suppose that $\chi_{I}$ is a fuzzy bi-interior ideal of the semigroup $M$. Then $I$ is a subsemigroup of $M$. We have

$$
\begin{aligned}
& \chi_{M} \circ \chi_{I} \circ \chi_{M} \cap \chi_{I} \circ \chi_{M} \circ \chi_{I} \subseteq \chi_{I} \\
\Rightarrow & \chi_{M I M} \cap \chi_{I M I} \subseteq \chi_{I} \\
\Rightarrow & \chi_{M I M \cap I M I} \subseteq \chi_{I}
\end{aligned}
$$

Therefore $M I M \cap I M I \subseteq I$.

Hence $I$ is a bi-interior ideal of the semigroup $M$.

Theorem 3.8. If $\mu$ and $\lambda$ are fuzzy bi-quasi ideals of a semigroup $M$, then $\mu \cap \lambda$ is a fuzzy bi-interior ideal of a semigroup $M$.

Proof. Let $\mu$ and $\lambda$ be fuzzy bi-interior ideals of the semigroup $M$. Then

$$
\begin{aligned}
\chi_{M} \circ \mu \cap \lambda(x) & =\sup _{x=a b}\left\{\min \left\{\chi_{M}(a), \mu \cap \lambda(b)\right\}\right\} \\
& =\sup _{x=a b}\left\{\min \left\{\chi_{M}(a), \min \{\mu(b), \lambda(b)\}\right\}\right. \\
& =\sup _{x=a b}\left\{\min \left\{\min \left\{\chi_{M}(a), \mu(b)\right\}, \min \left\{\chi_{M}(a), \lambda(b)\right\}\right\}\right. \\
& =\min \left\{\operatorname { s u p } _ { x = a b } \left\{\min \left\{\chi_{M}(a), \mu(b)\right\}, \sup _{x=a b}\left\{\min \left\{\chi_{M}(a), \lambda(b)\right\}\right\}\right.\right. \\
& =\min \left\{\chi_{M} \circ \mu(x) \cdot \chi_{M} \circ \lambda(x)\right\} \\
& =\chi_{M} \circ \mu \cap \chi_{M} \circ \lambda(x)
\end{aligned}
$$

Therefore $\chi_{M} \circ \mu \cap \lambda=\chi_{M} \circ \mu \cap \chi_{M} \circ \lambda$. 


$$
\begin{aligned}
\mu \cap \lambda \circ \chi_{M} \circ \mu \cap \lambda(x) & =\sup _{x=a b c}\left\{\min \left\{\mu \cap \lambda(a), \chi_{M} \circ \mu \cap \lambda(b c)\right\}\right\} \\
& =\sup _{x=a b c}\left\{\min \left\{\mu \cap \lambda(a), \chi_{M} \circ \mu \cap \chi_{M} \circ \lambda(b c)\right\}\right\} \\
& =\sup _{x=a b c}\left\{\min \left\{\min \{\mu(a), \lambda(a)\}, \min \left\{\chi_{M} \circ \mu(b c), \chi_{M} \circ \lambda(b c)\right\}\right\}\right. \\
& =\sup _{x=a b c}\left\{\min \left\{\min \left\{\mu(a), \chi_{M} \circ \mu(b c)\right\}, \min \left\{\lambda(a), \chi_{M} \circ \lambda(b c)\right\}\right\}\right. \\
& =\min \left\{\mu \circ \chi_{M} \circ \mu(x), \lambda \circ \chi_{M} \circ \lambda(x)\right\} \\
& =\mu \circ \chi_{M} \circ \mu \cap \lambda \circ \chi_{M} \circ \lambda(x) .
\end{aligned}
$$

Therefore $\mu \cap \lambda \circ \chi_{M} \circ \mu \cap \lambda=\mu \circ \chi_{M} \circ \mu \cap \lambda \circ \chi_{M} \circ \lambda$.

Similarly $\chi_{M} \circ \mu \cap \lambda \circ \chi_{M}=\chi_{M} \circ \mu \circ \chi_{M} \cap \chi_{M} \circ \lambda \circ \chi_{M}$.

Hence $\chi_{M} \circ \mu \cap \lambda \circ \chi_{M} \cap \mu \cap \lambda \circ \chi_{M} \circ \mu \cap \lambda=\left(\chi_{M} \circ \mu \circ \chi_{M}\right) \cap\left(\mu \circ \chi_{M} \circ \mu\right) \cap\left(\chi_{M} \circ \lambda \circ\right.$ $\left.\chi_{M}\right) \cap\left(\lambda \circ \chi_{M} \circ \lambda\right) \subseteq \mu \cap \lambda$.

Hence $\mu \cap \lambda$ is a fuzzy bi-interior ideal of the semigroup $M$.

Theorem 3.9. Let $\mu$ and $\lambda$ be a fuzzy right ideal and a fuzzy left ideal of a semigroup $M$ respectively. Then $\mu \cap \lambda$ is a fuzzy bi-interior ideal of the semigroup $M$.

Proofs of the following theorems are similar to theorems in [11 ]. So we omit the proofs.

Theorem 3.10. If $\mu$ is a fuzzy quasi-ideal of a regular semigroup $M$, then $\mu$ is a fuzzy ideal of the semigroup $M$.

Theorem 3.11. $M$ is a regular semigroup if and only $A B=A \cap B$, for any right ideal $A$ and left ideal $B$ of semigroup $M$.

Theorem 3.12. A semigroup $M$ is a regular if and only if $\lambda \circ \mu=\lambda \cap \mu$, for any fuzzy right ideal $\lambda$ and fuzzy left ideal $\mu$ of $M$.

Theorem 3.13. [12] Let $M$ be a semigroup. $M$ is a regular semigroup if and only if $B=$ $M B M \cap B M B$ for every bi-interior ideal of $M$.

Theorem 3.14. Let $M$ be a semigroup. Then $M$ is a regular if and only if $\mu=\chi_{M} \circ \mu \circ$ $\chi_{M} \cap \mu \circ \chi_{M} \circ \mu$, for any fuzzy bi-interior ideal $\mu$ of a semigroup $M$. 
Proof. Let $\mu$ be a fuzzy bi-interior ideal of the regular semigroup $M$.

Then $\chi_{M} \circ \mu \circ \chi_{M} \cap \mu \circ \chi_{M} \circ \mu \subseteq \mu$.

$$
\begin{aligned}
\chi_{M} \circ \mu \circ \chi_{M}(x) & =\sup _{x=x y x}\left\{\min \left\{\chi_{M}(x y), \mu(x)\right\}\right\}=\mu(x) \\
\mu \circ \chi_{M} \circ \mu(x) & =\sup _{x=x y x}\left\{\min \left\{\mu(x), \chi_{M} \circ \mu(y x)\right\}\right\} \\
& =\sup _{x=x y x}\left\{\min \left\{\mu(x), \sup _{y x=r s}\left\{\min \left\{\chi_{M}(r), \mu(s)\right\}\right\}\right\}\right\} \\
& =\sup _{x=x y x}\left\{\min \left\{\mu(x), \sup _{y x=r s}\{\min \{1, \mu(s)\}\}\right\}\right. \\
& \geq \sup _{x=x y x}\{\min \{\mu(x), \mu(x)\}\} \\
& =\mu(x) .
\end{aligned}
$$

Similarly, $\chi_{M} \circ \mu \circ \chi_{M} \subseteq \mu$. Therefore $\chi_{M} \circ \mu \cap \mu \circ \chi_{M} \circ \mu=\mu$.

Conversely suppose that $\mu=\chi_{M} \circ \mu \circ \chi_{M} \cap \mu \circ \chi_{M} \circ \mu$, for any fuzzy bi-interior ideal $\mu$ of the semigroup $M$. Let $B$ be a bi-interior ideal of the semigroup $M$.

Then by Theorem 3.7, $\chi_{B}$ be a fuzzy bi-interior ideal of the semigroup $M$.

$$
\text { Therefore } \begin{aligned}
\chi_{B} & =\chi_{M} \circ \chi_{B} \circ \chi_{M} \cap \chi_{B} \circ \chi_{M} \circ \chi_{B} \\
& =\chi_{M B M} \cap \chi_{B M B} \\
B & =M B M \cap B M B .
\end{aligned}
$$

By Theorem 3.13, $M$ is a regular semigroup.

Theorem 3.15. Let $M$ be a regular semigroup. Then $\mu$ is a fuzzy bi-interior ideal of $M$ if and only if $\mu$ is a fuzzy quasi ideal of $M$.

Proof. Let $\mu$ be a fuzzy bi-interior ideal of the semigroup $M$ and $x \in M$. Then

$$
\chi_{M} \circ \mu \circ \chi_{M} \cap \mu \circ \chi_{M} \circ \mu \subseteq \mu
$$

Suppose $\chi_{M} \circ \mu(x)>\mu(x)$.

Since $M$ is a regular, there exists $y \in M$ such that $x=x y x$.

$$
\text { Then } \begin{aligned}
\mu \circ \chi_{M} \circ \mu(x) & =\sup _{x=x y x}\left\{\min \left\{\mu(x y), \chi_{M} \circ \mu(x)\right\}\right) \\
& >\sup _{x=x y x}\{\min \{\mu(x), \mu(x)\}\} \\
& =\mu(x)
\end{aligned}
$$

Which is a contradiction. Therefore $\mu \circ \chi_{M} \cap \chi_{M} \circ \mu \subseteq \mu$.

By Theorem [3.10], converse is true 
Theorem 3.16. Let $M$ be a semigroup. Then $M$ is a regular if and only if $\mu \cap \gamma \subseteq$ $\gamma \circ \mu \circ \gamma \cap \mu \circ \gamma \circ \mu$, for every fuzzy bi-interior ideal $\mu$ and every fuzzy ideal $\gamma$ of a semigroup $M$.

Proof. Let $M$ be a regular semigroup and $x \in M$. Then there exists $y \in M$ such that $x=x y x$.

$$
\begin{aligned}
\mu \circ \gamma \circ \mu(x) & =\sup _{x=x y x}\{\min \{\mu \circ \gamma(x y), \mu(x)\}\} \\
& =\min \left\{\sup _{x y=x y x y}\{\min \{\mu(x), \gamma(y x y)\}, \mu(x)\}\right\} \\
& \geq \min \{\min \{\mu(x), \gamma(x)\}, \mu(x\} \\
& =\min \{\mu(x), \gamma(x)\}=\mu \cap \gamma(x) . \\
\gamma \circ \mu(x) & =\sup _{x=x y x}\{\min \{\gamma(x y), \mu(x)\}\} \\
& \geq \min \{\gamma(x), \mu(x)\}=\mu \cap \gamma(x) .
\end{aligned}
$$

Therefore $\mu \cap \gamma \subseteq \mu \circ \gamma \circ \mu$.Similarly we can prove $\mu \cap \gamma \subseteq \gamma \circ \mu \circ \gamma$. Hence $\mu \cap \gamma \subseteq \gamma \circ \mu \circ \gamma \cap \mu \circ \gamma \circ \mu$, Conversely suppose that the condition holds. Let $\mu$ be a fuzzy bi-interior ideal of the semigroup $M$.

$\mu \cap \chi_{M} \subseteq \chi_{M} \circ \mu \circ \chi_{M} \cap \mu \circ \chi_{M} \circ \mu$

$\mu \subseteq \chi_{M} \circ \mu \circ \chi_{M} \cap \mu \circ \chi_{M} \circ \mu$.

By Theorem 3.14, $M$ is a regular semigroup.

\section{Conclusion}

In this paper, we introduced the notion of a fuzzy bi-interior ideal in a semigroup. We studied some of their properties and characterized the regular semigroup in terms of fuzzy bi-interior ideal. In continuation of this paper, we propose to introduce the notion of a fuzzy bi-interior ideal of an ordered semiring and study the characterization of an ordered semiring in terms of a fuzzy bi-interior ideal.

\section{REFERENCES}

[1] R. A. Good and D. R. Hughes, Associated groups for a semigroup, Bull. Amer. Math. Soc., 58(1952), 624-625.

[2] K. Iseki, Quasi-ideals in semirings without zero, Proc. Japan Acad., 34 (1958), 79-84.

[3] K. Iseki, Ideal theory of semiring, Proc. Japan Acad., 32(1956), 554-559.

[4] R. D. Jagatap, Y.S. Pawar, Quasi-ideals and minimal quasi-ideals in $\Gamma$-semirings, Novi Sad J. Math., 39(2) (2009), 79-87.

[5] N. Kuroki, On fuzzy semigroups, Information Sciences, 53(3) (1991), 203-236.

[6] Lajos S. Szasz, On the bi-ideals in semigroups, Proc. Japan Acad., 45(1969), 710-712. 
[7] S. Lajos and F. A. Szasz, On the bi-ideals in associati ve ring, Proc. Japan Acad., 46 (1970), 505-507.

[8] W. Liu, Fuzzy invariant subgroups and fuzzy ideals, Fuzzy Sets Syst., 8 (2) (1982), 133-139.

[9] D. Mandal, Fuzzy ideals and fuzzy interior ideals in ordered semirings, Fuzzy Inf. Engg., 6 (2014), $101-114$.

[10] M. Murali Krishna Rao, T-fuzzy ideals in ordered $\Gamma$-semirings, Annals of fuzzy Math. Infor., 13(2) ( 2017), 253-276

[11] M. Murali Krishna Rao, Left bi-quasi ideals of semirings, Bull. Int. Math. Virtual Inst., 8(2018), 45-53.

[12] M. Murali Krishna Rao, Bi-quasi-ideals and fuzzy bi-quasi ideals of $\Gamma$ - semigroups, Bull. Int. Math. Virtual Inst., 7(2)(2017), 231-242.

[13] M. Murali Krishna Rao, $\Gamma$-semirings-I, Southeast Asian Bull. Math., 19 (1)(1995), 49-54.

[14] M. Murali Krishna Rao, $\Gamma-$ semiring with identity, Discuss. Math. Gen. Algebra Appl., 37 (2017) 189-207,

[15] M. Murali Krishna Rao, Ideals in ordered $\Gamma$-semirings, Discuss. Math. Gen. Algebra Appl., 38 (2018), $47-68$.

[16] M. Murali Krishna Rao, Bi-interior ideals in semigroups, Discuss. Math. Gen. Algebra Appl., 38 (2018), 69-78.

[17] M. Murali Krishna Rao, B. Venkateswarlu and N.Rafi, Left bi-quasi-ideals of $\Gamma$-semirings, Asia Pac. J. Math., 4(2) (2017), 144-153.

[18] M. Murali Krishna Rao and B. Venkateswarlu, Regular $\Gamma$-incline and field $\Gamma$-semiring, Novi sad. J. Math., 45(2) 2015, 155-171.

[19] M. Murali Krishna Rao, Fuzzy soft $\Gamma$-semiring and fuzzy soft $k$ ideal over $\Gamma$-semiring, Ann Fuzzy Math. Info., 9 (2)(2015), 12-25.

[20] M. Murali Krishna Rao, B. Venkateswarlu, $L-$ fuzzy ideals in $\Gamma$-semirings, Annals of fuzzy Math. Info., 10 (1) (2015), 1-16.

[21] M. Murali Krishna Rao,Fuzzy soft bi-ideal,fuzzy soft quasi-ideal and fuzzy soft interior ideal over ordered Г- semirings, Asia Pacific J. Math., 5(1)(2018), 60-84.

[22] M. Murali Krishna Rao, Fuzzy soft $\Gamma$-semiring and fuzzy soft $k$ ideal over $\Gamma$-semiring, Ann. Fuzzy Math. Info., 9 (2)(2015), 12-25.

[23] A. Rosenfeld, Fuzzy groups, J. Math. Anal.Appl. 35(1971), 512-517.

[24] A. M. Shabir , A Batod, A note on quasi ideal in semirings, Southeast Asian Bull. Math. 7(2004), 923-928.

[25] O. Steinfeld, Uher die quasi ideals, Von halbgruppend Publ. Math., Debrecen, 4 (1956), 262275.

[26] L. A. Zadeh, Fuzzy sets, Inf. Control, 8 (1965), 338-353. 